\title{
Effect of stiffness and thickness ratio of host plate and piezoelectric patches on reduction of the stress concentration factor
}

\author{
Javad Jafari Fesharaki ${ }^{1} \cdot$ Seyed Ghasem Madani $^{2} \cdot$ Sa'id Golabi $^{2}$
}

Received: 19 April 2015/ Accepted: 6 June 2016/Published online: 15 June 2016

(c) The Author(s) 2016. This article is published with open access at Springerlink.com

\begin{abstract}
This paper focuses on the effects of stiffness ratio and thickness ratio on reducing stress concentration factor using piezoelectric patches in a rectangular plate with a hole, as a classical shape. Various locations of actuators and induction of positive/negative strains into the host plate are investigated and the best location of patches is presented. The study investigated the ratio effects and piezoelectric patches bounded on a rectangular host plate having various thicknesses and materials. Results show that the best position of actuators varies based on values of thickness and stiffness ratios of the host plate and piezoelectric patches. Also, the location of maximum stress concentration is transmitted from top and bottom of the hole to another point around the edge by changing the location of the piezoelectric actuators. To verify the results, some experimental tests are applied. The results show good agreement between the finite element analysis and experimental tests.
\end{abstract}

Keywords Stress concentration factor Piezoelectric . Stiffness · Thickness

\section{Introduction}

In recent years, much progress has been made in smart structures. One of the high-performance structures that are frequently used includes piezoelectric materials. Many

Javad Jafari Fesharaki jafari@pmc.iaun.ac.ir

1 Department of Mechanical Engineering, Najafabad Branch, Islamic Azad University, Najafabad, Iran

2 Department of Mechanical Engineering, University of Kashan, Kashan, Iran studies on the properties for the use of piezoelectric materials such as controlling the vibration, buckling and stresses in structures have been developed by researchers.

Wang and Wu (2012) presented the active electromechanical properties of the piezoelectric material. They also represented the active mechanical-thermal properties of shape memory alloys and their application for structural repairing. Kang et al. (Kang and Tong 2008; Kang et al. 2012) investigated the simultaneous optimal distribution of material structure and a trilevel actuation voltage using topology optimization for a static shape control. Mehrabian and Yousefi-Koma (2011) introduced a new method to optimize the location of piezoelectric actuators for the vibration control of flexible structures. They investigated the vibration control of a flexible fin. Huang and Kim (2014) studied controlling the free-edge interlaminar stresses in composite plates using smart structures. They found that using piezoelectric actuators bonded on the surface or embedded in the composite laminates, stress significantly reduced in the plate. Also, location of piezoelectric actuators affects the stress distribution in composite laminates. Platz et al. (2011) studied reduction of crack propagation in aluminum panels using piezoelectric actuator near the crack tip by applying a compressive force to the panel surface. The use of a piezoelectric actuator repairing of a notched beam was investigated by $\mathrm{Wu}$ and Wang (2011). They used a small piezoelectric actuator placed near the cracks as a sensor to control stress concentration. Another piezoelectric was placed near the notch to reduce stress concentration. A new evolutionary algorithm for shape controlling of smart composite plates by applying piezoelectric patches was suggested by Nguyen et al. (Nguyen and Tong 2004, 2007; Nguyen et al. 2007). They were able to optimize the electric field and obtain the location and size of piezoelectric actuators for the desired 
structures. Hsu et al. (2014) studied energy harvesting by piezoelectric actuators from kinetic energy structure, such as structural vibrations to convert the electrical energy. Buckling control of rigid panels using piezoelectric actuators was presented by Sridharan and Kim (2009).They applied piezoelectric actuators on the surface of the panel to prevent buckling. Using a piezoelectric actuator, Franco Correia et al. (2003) investigated increasing buckling load of a laminated composite plate. Chee et al. (2002) and Lin and Nien (2007) worked on static shape control of composite plates. They examined the effects of the piezoelectric actuator and its place in shape control. Zhang et al. (2000) used the genetic algorithm for optimal control of flexible smart structures and optimization of piezoelectric actuator placement in a desired structure. A semi-analytical solution for static and dynamic analysis of a laminated aluminum plate that was attached by piezoelectric patches was provided by Qing et al. (2006). Using the genetic algorithm, Da Mota Silva et al. (2004) studied the shape control of composite plates using piezoelectric patches. They described the behaviors of the structure and piezoelectric actuator using finite element model. To harvest energy, Nakasone and Silva (2010) worked on developing topology optimization formulation for a dynamic design of laminated piezoelectric plates. Rafiee et al. (2014) also developed a study for the purpose of energy harvesting. They worked on nonlinear analysis of piezoelectric functionally graded carbon nanotubes in mechanical and thermal loading. Using the genetic algorithm, Roy and Chakraborty (2009) studied the optimal vibration control of a smart composite shell. They formulated finite elements layered shell for coupled electromechanical analysis of a composite structure integrated with piezoelectric patches. $\mathrm{Wu}$ and Wang (2010) investigated a method for repairing the vibrating delaminated beam structures using piezoelectric actuators. Kumar et al. (2008) presented a finite element formulation for dynamic and static responses of a laminated composite shell, including piezoelectric actuators subjected to thermal, mechanical and electric loads. Quintero et al. (2014) investigated the optimization design through modeling a thinned bulk-PZT-based vibration energy harvester on a flexible polymeric substrate. Kurata et al. (2013) studied a method to find damages of a sensing system by monitoring seismic damage in steel structures. Jadhav and Bajoria (2013) studied forced and free vibration analysis of functionally graded materials. They used piezoelectric actuator at the top and bottom to control the vibration of the plate. Sensharma et al. (Sensharma et al. 1993; Sensharma and Haftka 1996) worked on reducing stress concentration in a plate with a hole using piezoelectric actuators. Their aim was to achieve maximum reduction in stress concentration in the plate using piezoelectric patches near the hole. The use of piezoelectric patches to reduce the stress concentration factor in a plate with a hole was presented by Shah et al. (1994). They showed that if the piezoelectric patches were placed in an area bearing a high stress, the stress in the plate would be decreased while the stress in the piezoelectric patches would be increased. They proposed that locating actuators in the compression area and inducing positive strain in a host plate, the stress flows through the host plate are changed and consequently lead to reducing stress in the plate indirectly.

In this paper, two methods of reduction in stress concentration factor using piezoelectric patches are discussed. In the first method, inducing positive strain to the host plate results in the best position of locating the piezoelectric patches. For the second method, negative strains are induced in the host plate and the stress line is redistributed to reduce the stress concentration factor. For these two methods, the effects of stiffness ratio and thickness ratio of the host plate and piezoelectric patches are investigated. Some experimental tests are considered to validate the results.

\section{Finite-element formulation}

Mechanical behaviors of a thin plate under tension are modeled by first-order shear deformation theory. The displacement components $u, v$ and $w$ at any point of plate elements are assumed to be:

$$
\begin{aligned}
& u(x, y, z)=u_{0}(x, y)+z \theta_{x}(x, y) \\
& v(x, y, z)=v_{0}(x, y)+z \theta_{y}(x, y) \\
& w(x, y, z)=w_{0}(x, y)
\end{aligned}
$$

where $u_{0}, v_{0}$ and $w_{0}$ are the mid plane displacements and $\theta_{x}$ and $\theta_{y}$ are the rotations around $x$ and $y$ axes. Using isoperimetric relationships, the displacements and coordinates inside the element are defined as

$u=\sum_{i=1}^{n} N_{i} \delta_{i}, x=\sum_{i=1}^{n} N_{i} x_{i}, y=\sum_{i=1}^{n} N_{i} y_{i}$

where $N_{i}$ is the element shape functions and $n$ is the number of element nodes. The constitutive relations for the piezoelectric material can be expressed as

$\sigma=Q \varepsilon-e E, D=e^{T} \varepsilon+p E$,

where " $\sigma$ " is the stress vector, " $\varepsilon$ " the strain vector, " $Q$ " the elastic stiffness matrix of the plate element, " $e$ " the piezoelectric stress matrix, " $p$ " the permittivity matrix and " $D$ " the electric displacement vector. The electric field vector " $E$ " is defined as

$E=-\Delta \Phi$,

where " $\Phi$ " is the electric voltage applied across the thickness of piezoelectric patches. 
Table 1 Seven conditions for stiffness ratio and thickness ratio

\begin{tabular}{llllllll}
\hline Condition number & No. 1 & No. 2 & No. 3 & No. 4 & No. 5 & No. 6 & No. 7 \\
\hline Stiffness ratio (Rs) & $\mathrm{Rs}=1 / 4$ & $\mathrm{Rs}=1 / 3$ & $\mathrm{Rs}=1 / 2$ & $\mathrm{Rs}=1$ & $\mathrm{Rs}=2$ & $\mathrm{Rs}=3$ & $\mathrm{Rs}=4$ \\
Thickness ratio (Rt) & $\mathrm{Rt}=1 / 4$ & $\mathrm{Rt}=1 / 3$ & $\mathrm{Rt}=1 / 2$ & $\mathrm{Rt}=1$ & $\mathrm{Rt}=2$ & $\mathrm{Rt}=3$ & $\mathrm{Rt}=4$ \\
\hline
\end{tabular}

To solve the equation of a plate motion containing piezoelectric patches, the Hamilton's principle is used. For an electromechanical coupled system, the principle is:

$\int_{a 1}^{a 2} \delta\left(T-U+W_{\mathrm{ext}}\right) d a=0$,

where " $a_{1}$ " and " $a_{2}$ " are arbitrary instants and " $T$ ", " $U$ " and " $W_{\text {ext }}$ " kinetic energy, potential energy and the work done by electrical and external forces and, respectively. These parameters are defined as:

$$
\begin{aligned}
& T=\int_{V} \frac{1}{2} \rho\{\dot{S}\}^{T}\{\dot{S}\} \mathrm{d} V, \\
& U=\int_{V} \frac{1}{2}\left[\{\varepsilon\}^{T}\{\sigma\}-\{E\}^{T}\{D\}\right] \mathrm{d} V, \\
& W_{\text {ext }}=\sum_{i=1}^{m_{\mathrm{f}}}\{S\}^{T}\left\{F_{\mathrm{b}}\right\},
\end{aligned}
$$

where, " $\rho$ ", " $F_{\mathrm{b}}$ " and " $m_{\mathrm{f}}$ " are mass density, external force vector and number of applied forces, respectively, and " $V$ " is the volume of the structure.

\section{Model and problem definition}

Two methods for controlling the stress in a plate with hole were provided previously (Shah et al. 1994). In the first proposed method, stress is directly decreased in the plate by placing piezoelectric actuators at the points bearing high stress, and by applying positive strain on the host plate. The problem of this method is that as the stress concentration factor reduces in the host plate, the stress increases in the piezoelectric actuators. In the second method, by redistributing the stress flow line in the host plate using piezoelectric actuators and by creating a negative strain, the stress in the host plate is reduced indirectly.

In previous researches, the effect of thickness and stiffness ratio of piezoelectric actuators and the host plate on the location of piezoelectric patches and reduction in stress concentration factor has not been studied. However, the thickness ratio and stiffness ratio of piezoelectric patches and host plate effect on the location of piezoelectric patches and the decrement rate of stress concentration factor. Stiffness ratio (Rs) and thickness ratio (Rt) of the piezoelectric patches and plate are defined as follows:

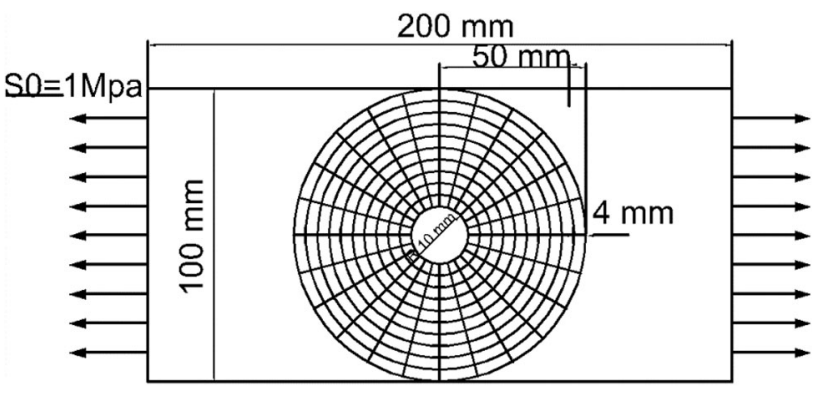

Fig. 1 The geometry of plate and grid mesh

Stiffness ratio $(\mathrm{Rs})=\frac{(E)_{\text {Plate }}}{\left(E_{0}\right)_{\text {Piezo }}}$,

Thickness ratio $(\mathrm{Rt})=\frac{(t)_{\text {Plate }}}{\left(t_{0}\right)_{\text {Piezo }}}$.

To investigate the two proposed methods and analyze the effects of stiffness ratio and thickness ratio of the host plate and the piezoelectric patches on locating the piezoelectric actuator and reducing the stress concentration factor around the hole, seven conditions listed in Table 1 are considered.

For the host plate, a thin rectangular plate subjected to uniform tension $1 \mathrm{Mpa}$ is considered. The geometry of the plate is presented in Fig. 1. Because of accruing the stress concentration around the hole to achieve the maximum reduction in stress concentration factor, piezoelectric actuators should be placed near the hole. Therefore, a virtual grid mesh near the hole for the location of the piezoelectric patches as a circle sector with a $15^{\circ}$ radius and a $4 \mathrm{~mm}$ width is considered. The piezoelectric patches are polarized in the thickness direction. To simulate and analyze the mentioned problem, a python code is developed. In finite element formulation, the element "C3D20RA 20-node quadratic brick" is considered for plates and elements "C3D20RE-A 20-node quadratic piezoelectric brick" is considered for piezoelectric parts.

\section{Effect of stiffness and thickness ratio on piezoelectric location}

- Method 1. Piezoelectric actuators reduce the stress concentration factor directly by creating a positive strain in the host plate.

To find the best location for piezoelectric patch placements, after finding the optimal voltage for each location of 

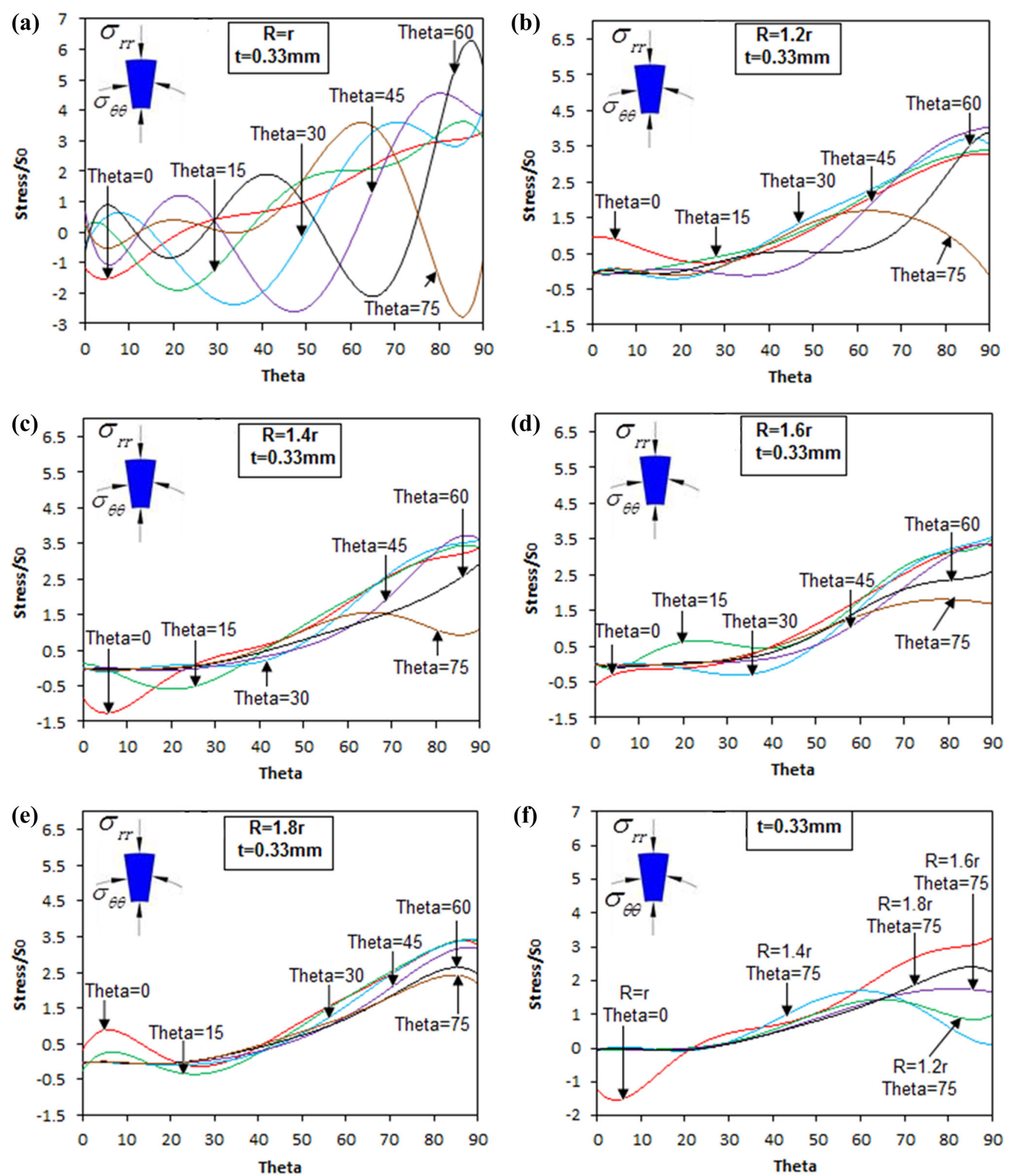

Fig. 2 Longitudinal stress around the hole in the host plate for Rt $=1 / 3$ (condition 2)

piezoelectric patches in the grid mesh presented in Fig. 1, the maximum stress around the hole is obtained as presented in Fig. 2.
To discuss the best location, Condition Number 2 of Table 1 is selected. Figure 2 shows the longitudinal stress around the hole in the host plate. Figure 2a shows the stress 
curves for the case in which piezoelectric actuators are located in the first row of the grid mesh. According to this figure, it can be seen that all curves are maintained from the beginning to the end as sinusoidal curves. Curve (theta $=75$ ) at $90^{\circ}$ angles, comparing other curves has the lowest stress, and consequently reduces the maximum stress concentration factor. Curve (theta $=60$ ) is located at $90^{\circ}$ higher than the other curves, resulting in minimum reduction in the stress concentration factor.

The curve (theta $=75$ ) has the maximum reduction in the stress concentration factor; however, at a range of point $60^{\circ}$, high stress can be observed.

As the purpose is reducing the stress concentration factor at all points of the plate, it can be seen that the curve (theta $=0$ ) has a mild shape from the beginning to the end and results in the best curve for reducing the stress concentration factor. Therefore, the piezoelectric patches should be placed at this point to achieve the maximum reduction in stress concentration factor at all points of the plate.

The curves presented in Fig. $2 b$ show the stress related to the location of piezoelectric actuators in the second row of the grid mesh. It can be seen that the curves change to a smooth shape from a sinusoidal shape as shown in Fig. 2a. Figure 2c-e demonstrate the stresses in the plate for placement of piezoelectric actuators at the third, fourth and fifth rows of the grid mesh, respectively. These figures show that the curve (theta $=75$ ) in all figures has the lowest stress at $90^{\circ}$. Figure $2 \mathrm{f}$ represents a comparison of the discussed results for the best location of the patches. Also, the curve $(R=1.4 \mathrm{r}$, theta $=75)$ has the lowest stress at the $90^{\circ}$ angle and the curve $(R=1.2 \mathrm{r}$, theta $=75)$ has a lower stress concentration factor around the hole. According to Fig. 2, the best location for the placement of piezoelectric actuators to achieve the maximum reduction in the stress concentration factor is at the top and bottom of the hole, at a distance of $0.2 \mathrm{~mm}$ from its edge. The best location of piezoelectric patches in the mesh grid is illustrated in Fig. 3.

The longitudinal stress around holes in the host plate for the best location of the piezoelectric patch for Rs $\leq 1$ is presented in Fig. 4. As it is clear, the stress curves start from zero value at $0^{\circ}$ and increase around the hole as the angle increases.

Figure 5 shows the comparison of longitudinal stresses around the hole in the host plate for the best location of piezoelectric actuators placement for (Rs $\geq 1$ ). Curves have a peak at $65^{\circ}$ and meet together at $72^{\circ}$. The curve $(E /$ $E 0=1)$ at $90^{\circ}$ has the highest stress and results in a minimal reduction in the stress concentration factor. Curve

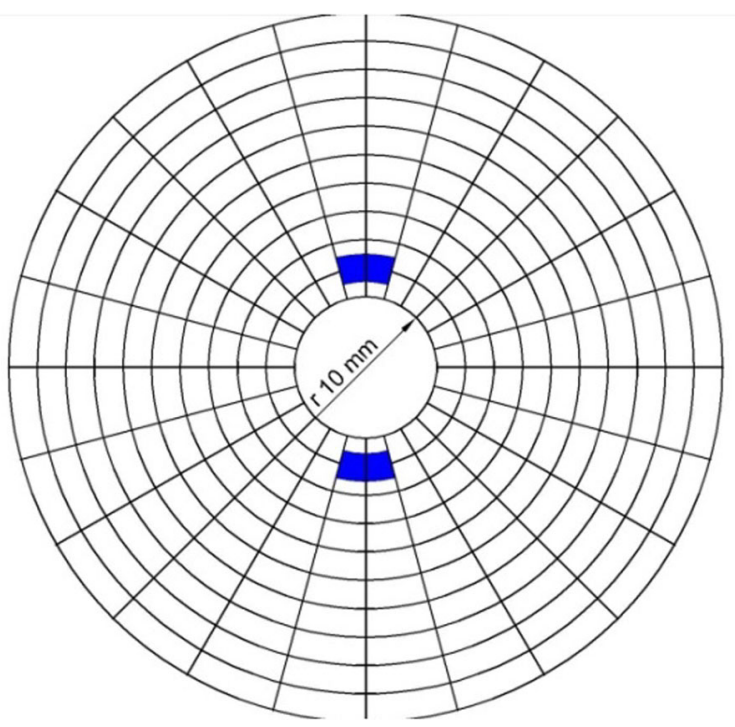

Fig. 3 The optimal location of piezoelectric patches

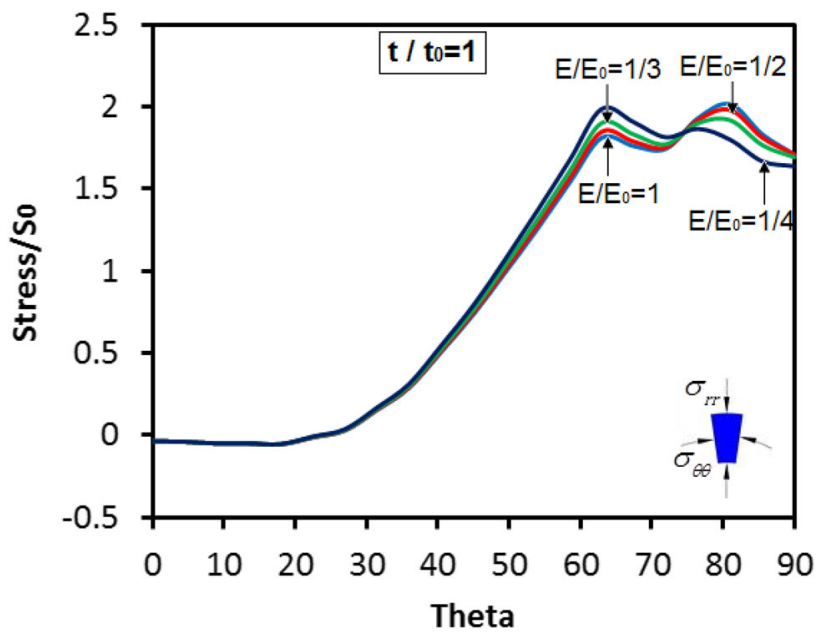

Fig. 4 Longitudinal stress in the host plate for Rs $\leq 1$

$(E / E 0=4)$ has the greatest reduction in the stress concentration factor, as it has the lowest stress at $90^{\circ}$.

The results obtained from two previous figures have been illustrated in Fig. 6. The reduction in the stress concentration factor for different stiffness ratios is presented in Fig. 6. It shows that $E / E 0=1 / 4$ has the greatest reduction in the stress concentration factor. As a result, piezoelectric actuators in this case have a greater ability to reduce the stress concentration factor. Accordingly, to achieve maximum reduction in the stress concentration factor, the host plate material should be softer than that of the piezoelectric actuator. 


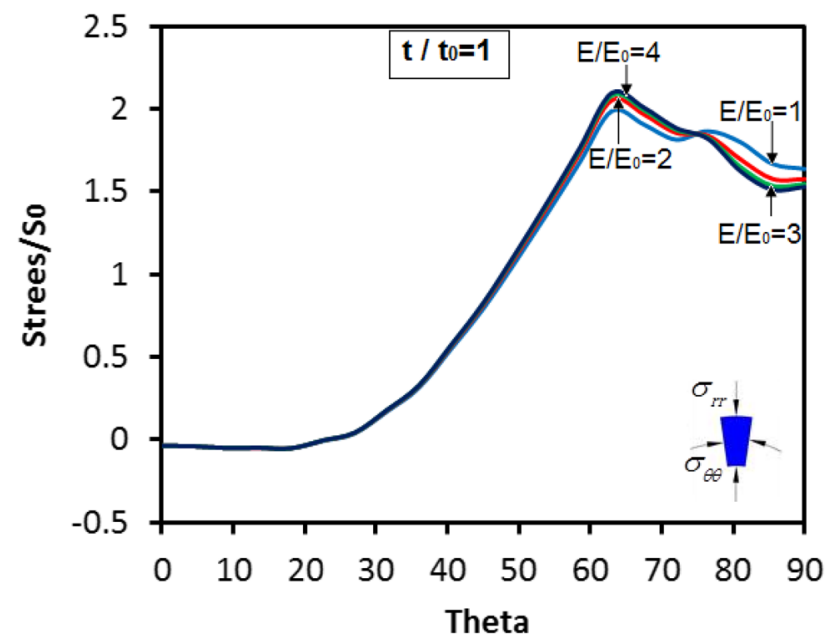

Fig. 5 Longitudinal stress in the host plate for Rs $\geq 1$

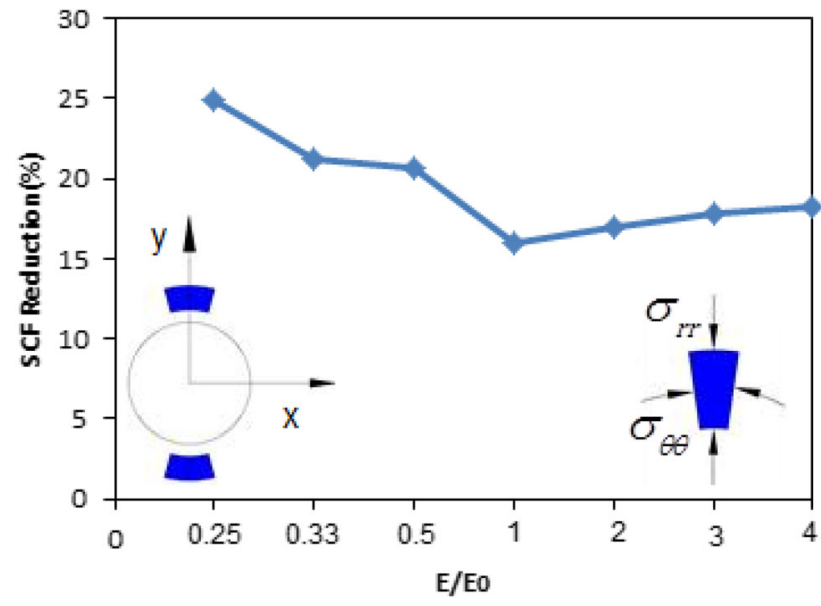

Fig. 6 Maximum reduction in stress concentration factor for various Rs

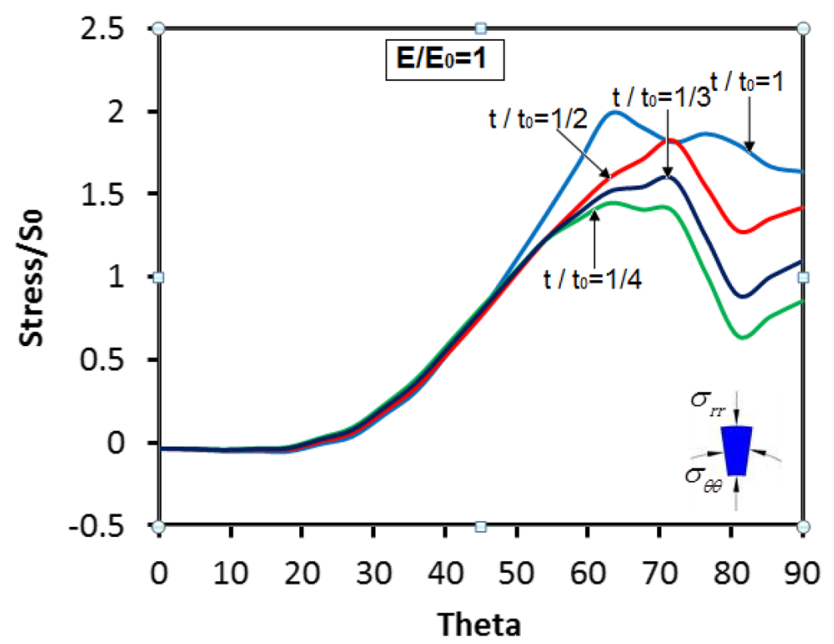

Fig. 7 Longitudinal stress in the host plate for Rt $\leq 1$

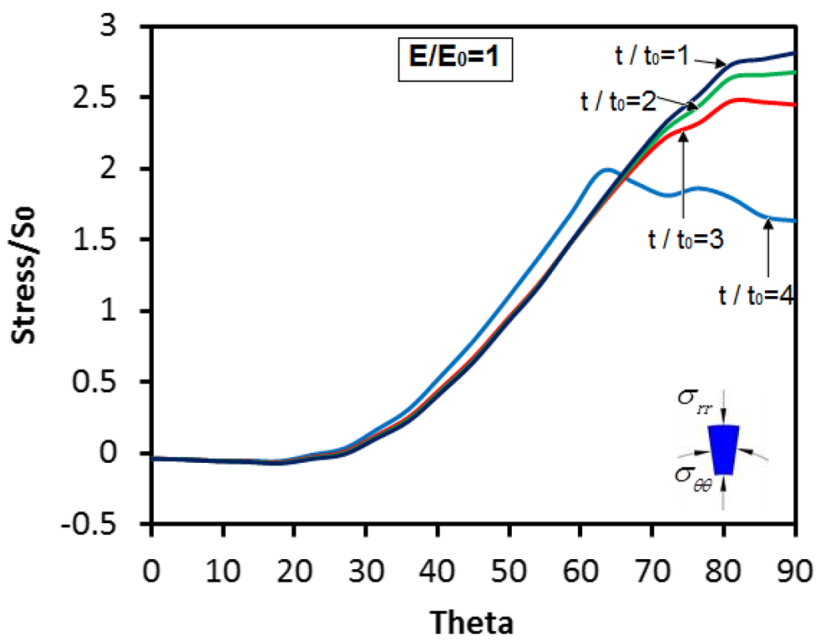

Fig. 8 Longitudinal stress in the host plate for $\mathrm{Rt} \geq 1$

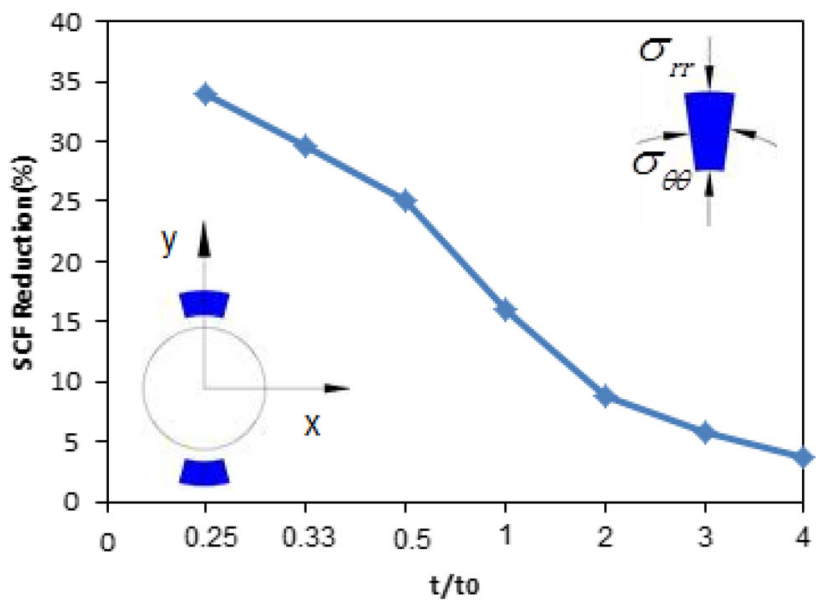

Fig. 9 Maximum stress concentration reduction for the various "Rt"

Figure 7 shows longitudinal stress around the hole on the host plate for a thickness ratio smaller than one. Curve $t / t_{0}=1$ at $63^{\circ}$ has a peak and in the end point for $90^{\circ}$ has a higher stress than other curves and leads to smaller reduction in the stress concentration factor. The curve $t /$ $t_{0}=1 / 4$, at $90^{\circ}$ has a minimum stress and leads to maximum reduction in the stress concentration factor. Figure 8 shows the longitudinal stresses around the hole for thickness ratios larger than unity. It is clear that the curve $t /$ $t_{0}=4$ at $90^{\circ}$ has the minimum stress and results in a maximum reduction in the stress concentration factor.

Figure 9 shows the results for stress concentration reduction in various thickness ratios. $t / t_{0}=1 / 4$ causes the maximum reduction in stress concentration factor and the $t /$ $t_{0}=4$ has minimum reduction, as Fig. 9 shows. Therefore, to achieve a maximum reduction in the stress concentration 

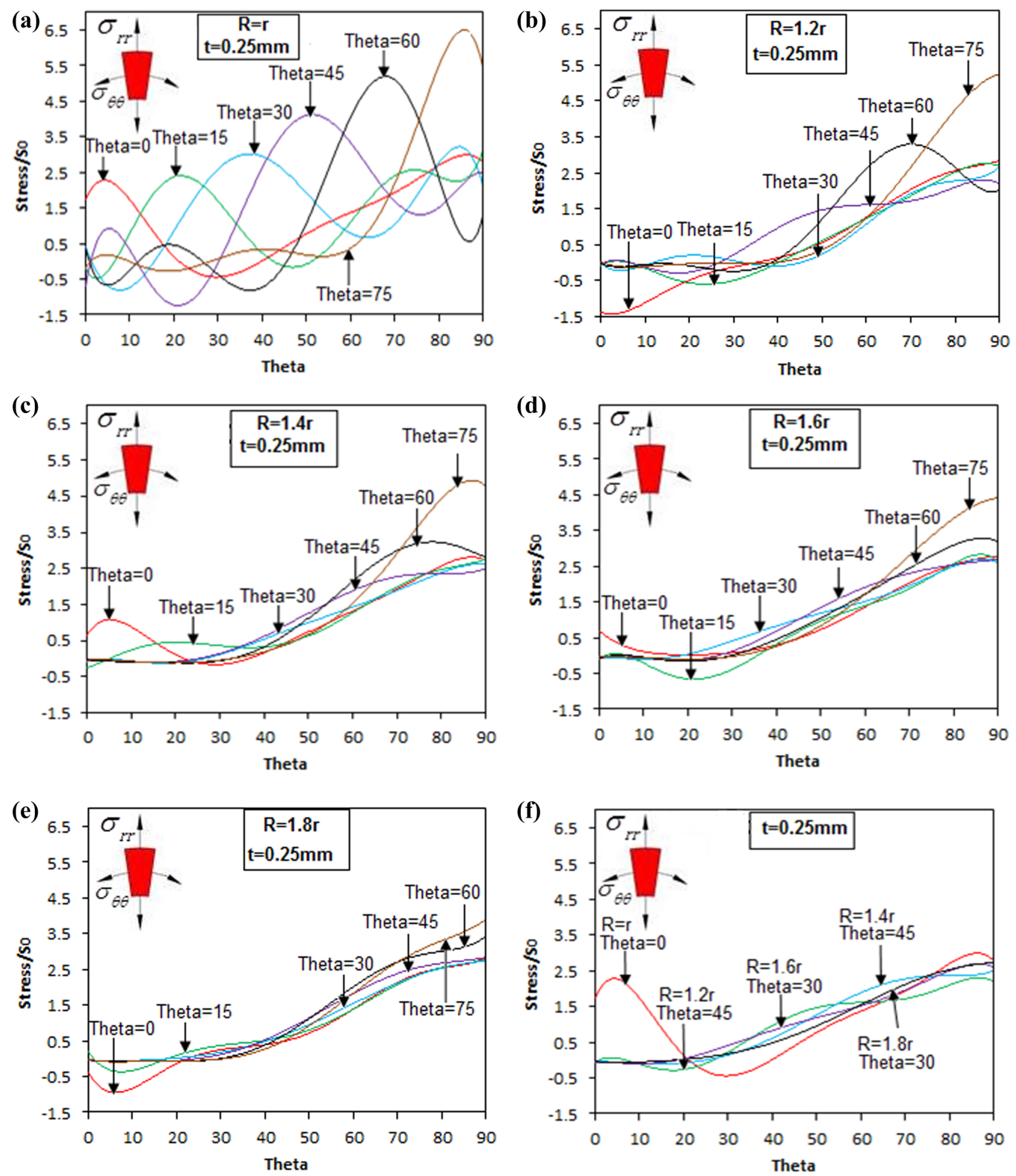

Fig. 10 Longitudinal stress around hole in the host plate $(\mathrm{Rs}=1 / 4$, condition 1$)$ 
Fig. 11 Optimal placement of piezoelectric patches for $\mathrm{Rs} \leq 1$
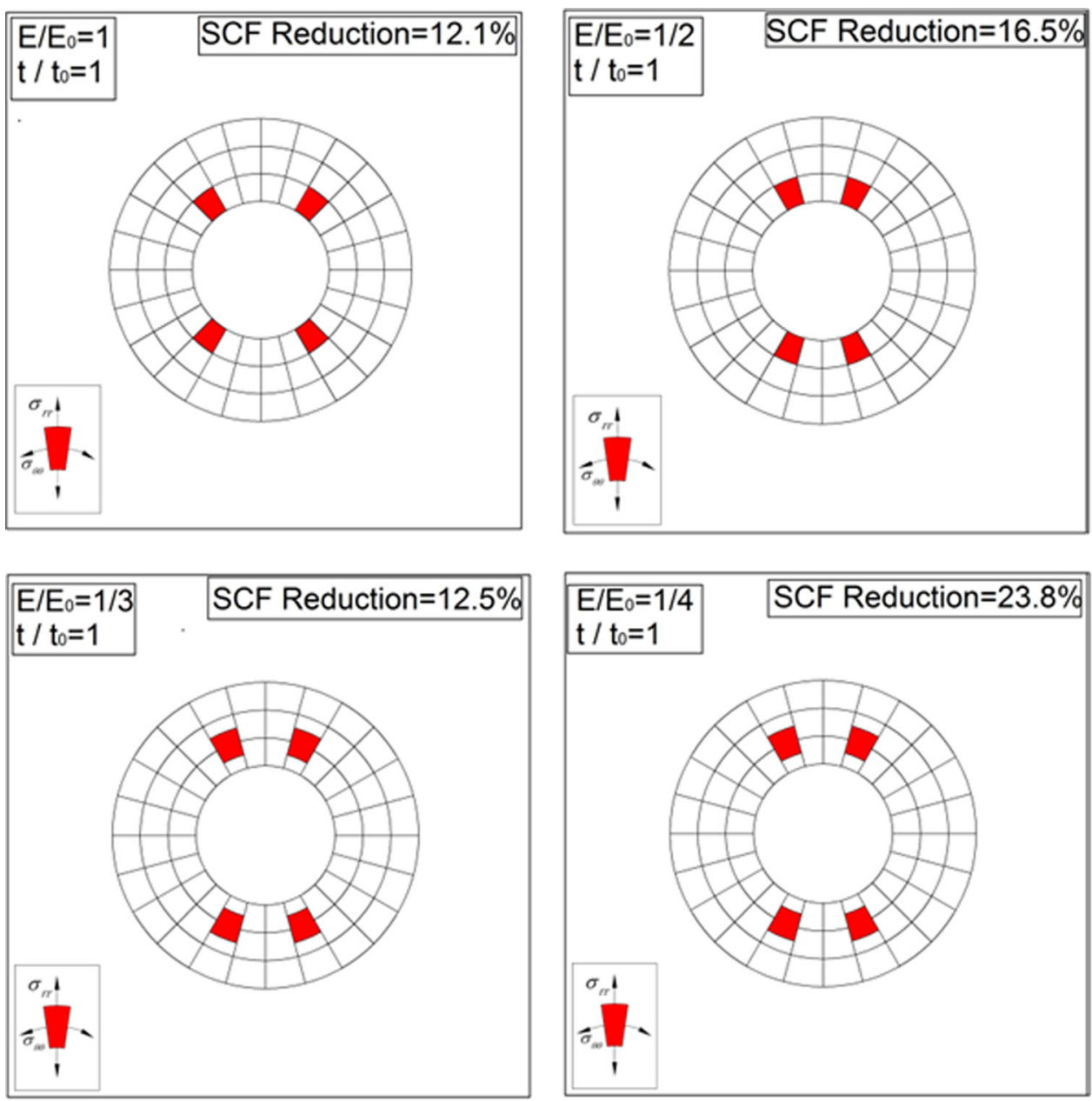

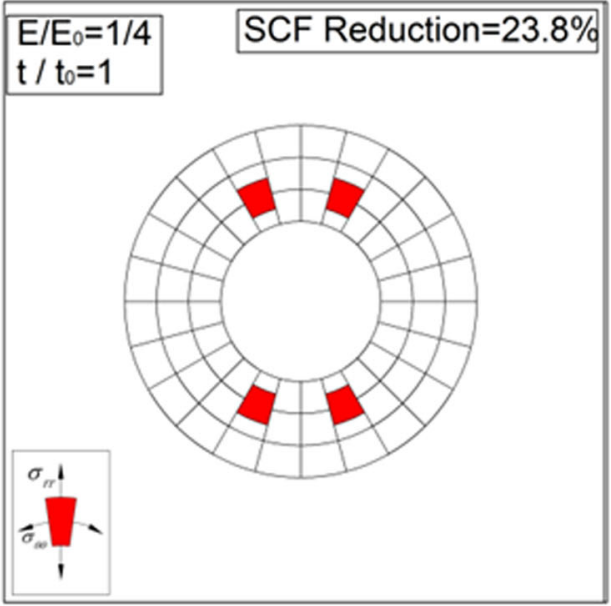

factor, the plate thickness should be lower than that of the piezoelectric actuator.

- Method 2. For creating negative strain in the host plate, piezoelectric actuators redistribute the stress flow line and reduce the stress concentration factor indirectly.

To show the results of the best location of piezoelectric actuators, condition number No. 1 of Table 1 is selected.

Figure 10 shows the longitudinal stress around the hole in the host plate. Figure 10a shows the stress curves for a case in which piezoelectric actuators are located in the first row of the grid mesh. All curves are sinusoidal. The curve for theta $=75$ at $90^{\circ}$ has higher stress than the others. This curve (theta $=75$ ) causes a maximum reduction in the stress concentration factor, but high stress can be observed around $68^{\circ}$. Because the purpose of using piezoelectric patches is reducing stress concentration factor in all points of the plate, the curve (theta $=0$ ) has a mild shape from the beginning to the end. As a result, the curve theta $=0$ is the best curve of the reduction of the stress concentration factor. Therefore, the piezoelectric patches should be placed at this point, to achieve the maximum reduction in stress concentration factor at all points of the plate.

Curves presented in Fig. 10b are related to the location of piezoelectric actuators in the second row of the grid mesh. The curve theta $=60$ at $90^{\circ}$ angles has the minimum value of stress and results in the maximum reduction in the stress concentration factor. For the curve, however, the stress has high value at $70^{\circ}$ angles.

Curves presented in Fig. 10c show the longitudinal stress around the hole in which piezoelectric actuators are located at the third row of the mesh grid. It can be seen that curve theta $=45$ at $90^{\circ}$ has a minimum value of stress and causes a maximum reduction in the stress concentration 
Fig. 12 Optimal placement of piezoelectric patches for $R s \geq 1$
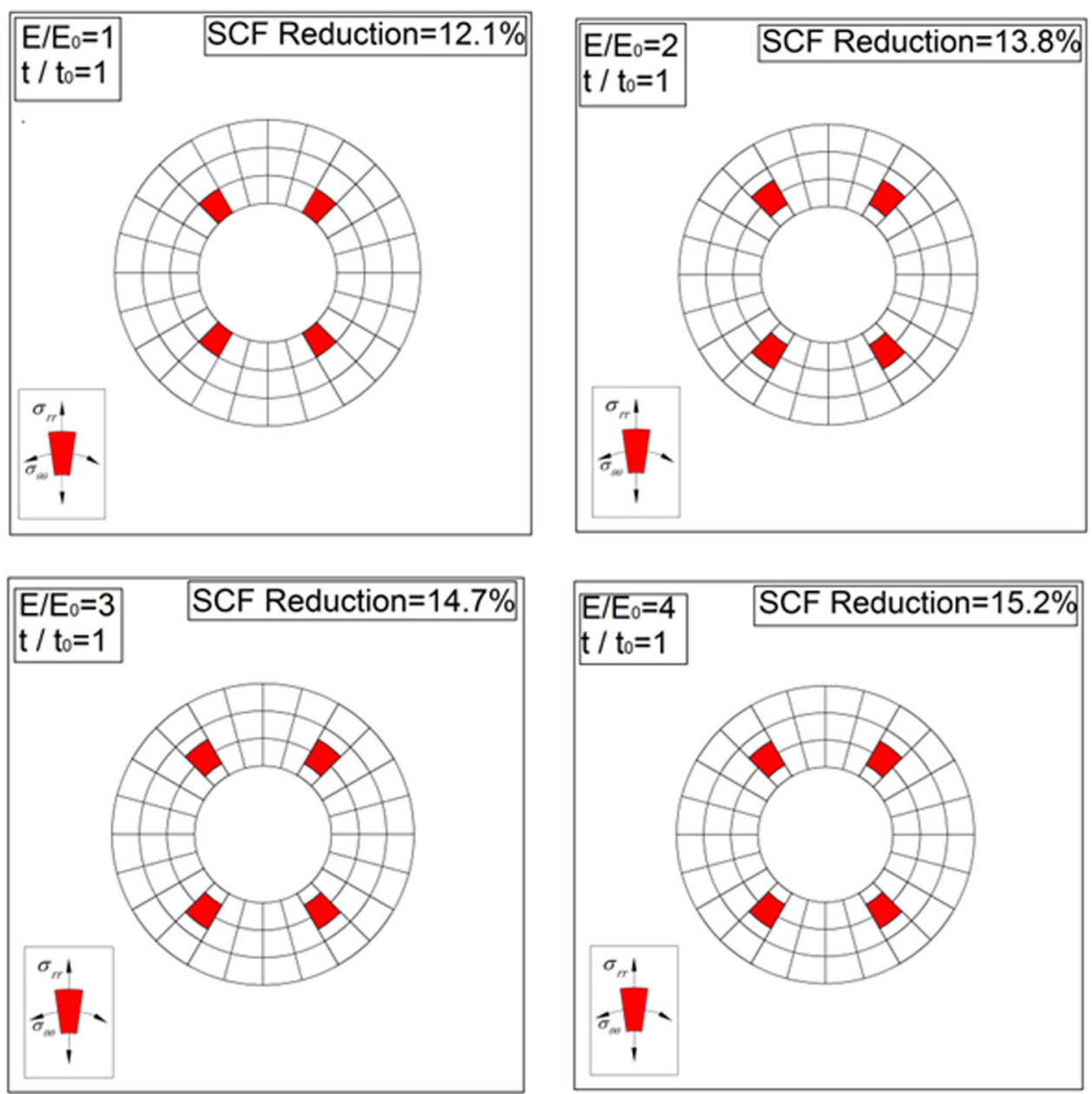

factor. Figure 10d, e demonstrates the placement of piezoelectric actuators in the fourth and fifth rows of the mesh grid, respectively.

Figure 10f provides the comparison of the best mentioned results. It shows that curves $R=1.2 \mathrm{r}$, theta $=45$ at $90^{\circ}$ have minimum stress and therefore have a maximum reduction in stress concentration factor. Then, the piezoelectric actuators should be placed at this point.

In Fig. 11, the optimal placement of piezoelectric patches for $\mathrm{Rs} \leq 1$ is presented. For $\mathrm{Rs}=1$ to $\mathrm{Rs}=1 / 4$, the piezoelectric patches are placed around the hole for $\mathrm{Rs}=1$ and $\mathrm{Rs}=2$. However for others, the patches are located neither at the top nor at the bottom of the hole. Figure 12 presented the optimal placement of piezoelectric patches for $\mathrm{Rs} \geq 1$. For this condition also, the piezoelectric patches are located neither at the top nor at the bottom of the hole. The optimal placement of the patches are at distances from the edge of the hole.
In Fig. 13, the optimal placement of piezoelectric patches for $\mathrm{Rt} \leq 1$ is presented. For $\mathrm{Rt}=1$ to $\mathrm{Rt}=1 / 4$, piezoelectric patches are located at the same angle and the distance between the patches and the edge of the hole is $0.2 \mathrm{~mm}$.

Optimal placement of piezoelectric patches for $\mathrm{Rt} \geq 1$ is presented in Fig. 14. For $\mathrm{Rt}=1$ to $\mathrm{Rt}=4$, the piezoelectric patches are located at the edge of the hole and neither at the top nor at the bottom. Another point from Fig. 14 is that the thickness ratio and stiffness ratio of the host plate and piezoelectric patches may affect the location of patches around the hole.

The longitudinal stress around holes in the host plate for the best location of the piezoelectric patch for $\mathrm{Rs} \leq 1$ and $\mathrm{Rs} \geq 1$ are shown in Figs. 15 and 16, respectively. Figure 15 shows that the $\mathrm{Rs}=1 / 4$ has minimum reduction in stress concentration factor in the host plate. It is noticeable that although the stress at $90^{\circ}$ is reduced for $\mathrm{Rs}=1 / 4$, the stress in another point around the hole 
Fig. 13 Optimal placement of piezoelectric patches for $\mathrm{Rt} \leq 1$
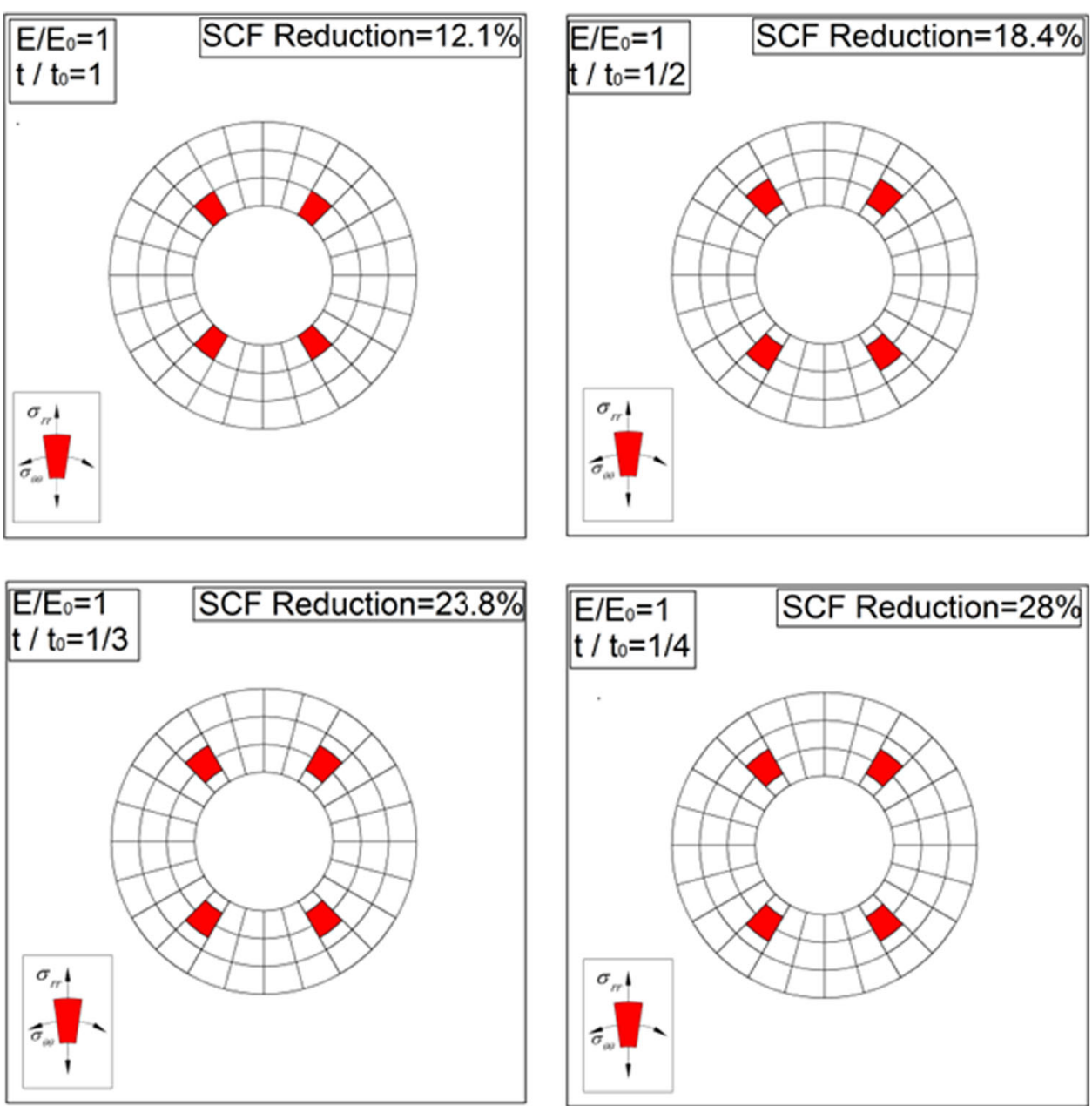

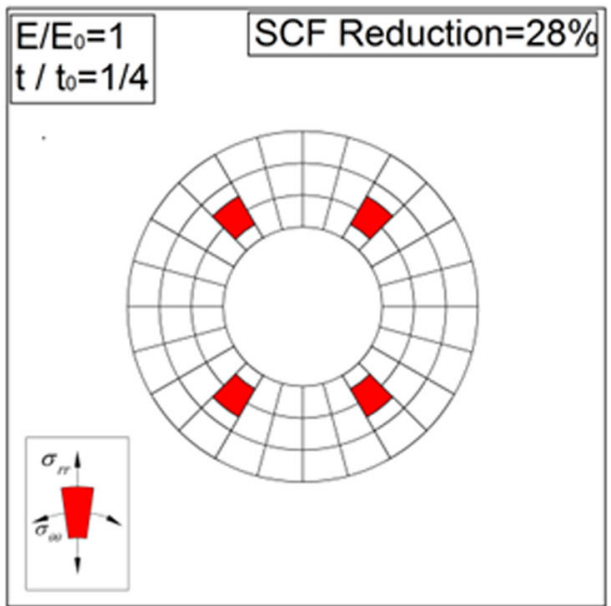

is increased. It means that the maximum stress from the top and bottom of the hole is transmitted to another point around the hole.

The results obtained from two previous figures have been illustrated in Fig. 17. In this figure, the reduction of the stress concentration factor for different stiffness ratios is presented. The results show that the $E / E_{0}=1 / 4$ has the greatest reduction in the stress concentration factor. As a result, the piezoelectric actuators in this case have a greater capability to reduce the stress concentration factor. To achieve the maximum reduction in the stress concentration factor in the host plate, the host plate material should be softer than that of the piezoelectric actuator.

Figures 18 and 19 show the longitudinal stress around the hole in the host plate for thickness ratio smaller and greater than unity, respectively. As these figures show for thickness ratio smaller than one, $t / t_{0}=1 / 4$ has minimum stress around the hole.
Figure 20 shows the results obtained from Figs. 18 and 19. In Fig. 20, the reduction of the stress concentration factor for different thickness ratios is presented. It can be seen that the curve $t / t_{0}=1 / 4$ has the maximum reduction and $t / t_{0}=4$ has the minimum reduction in the stress concentration factor. To achieve the maximum reduction in the stress concentration factor, the plate thickness should be lower than that of the piezoelectric actuators.

\section{Validating results}

To validate the results, some experimental tests are applied. The geometry of the plate and piezoelectric patches are the same, as mentioned previously. The host page is located under tension $1 \mathrm{Mpa}$. To induce positive strain in the host plate by piezoelectric actuators, $\mathrm{Rs}=1 / 2$ is selected and to induce negative strain in the host plate $\mathrm{Rt}=2$ is selected. To specify the effect of the stiffness ratio and the thickness 
Fig. 14 Optimal placement of piezoelectric patches for $\mathrm{Rt} \geq 1$
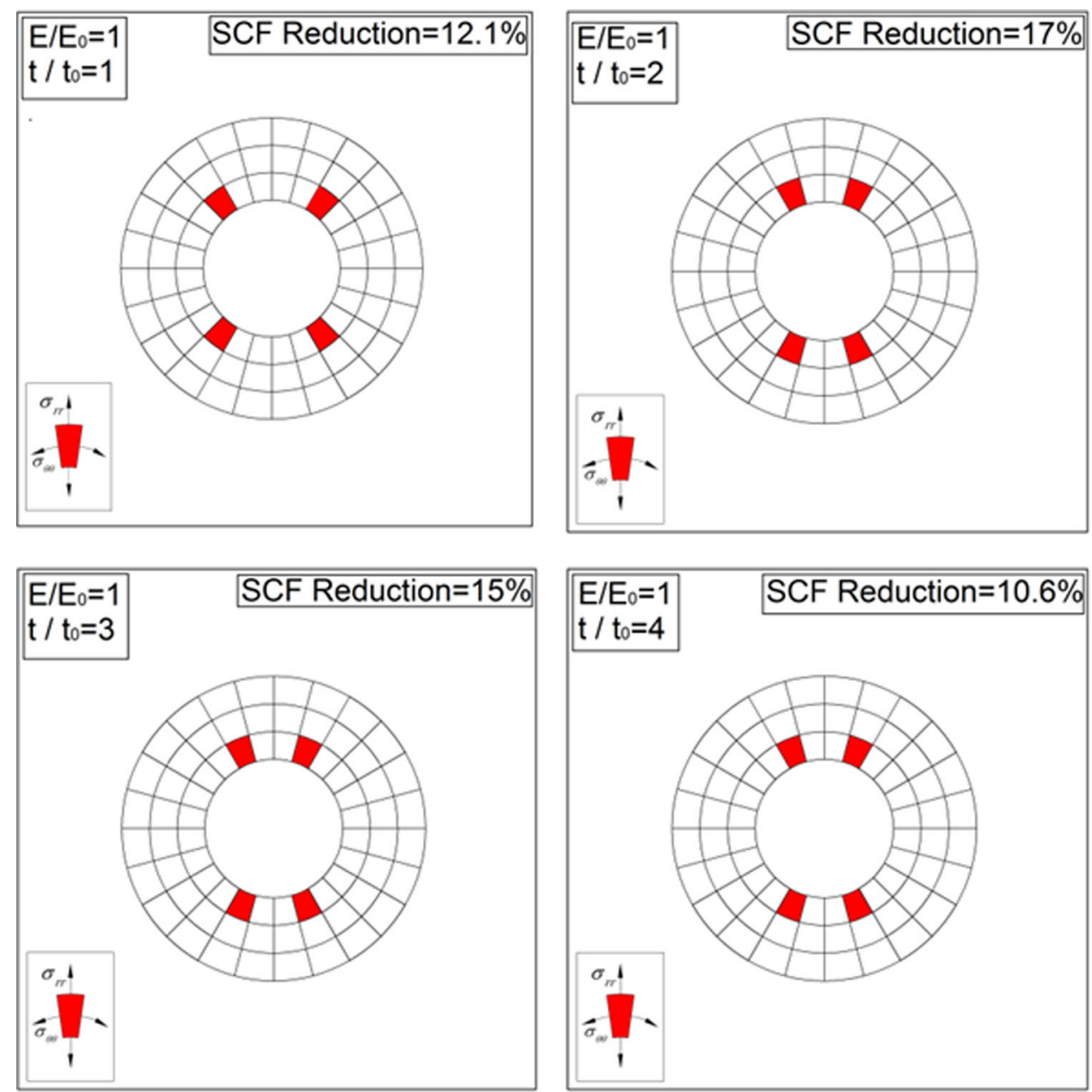

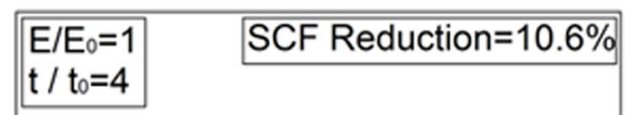

$\sigma_{n}$

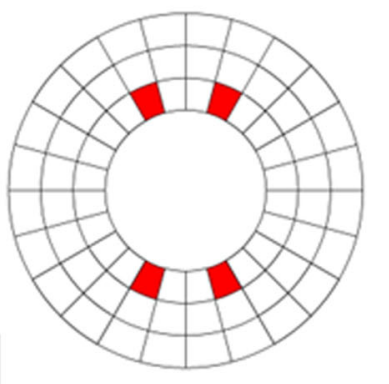

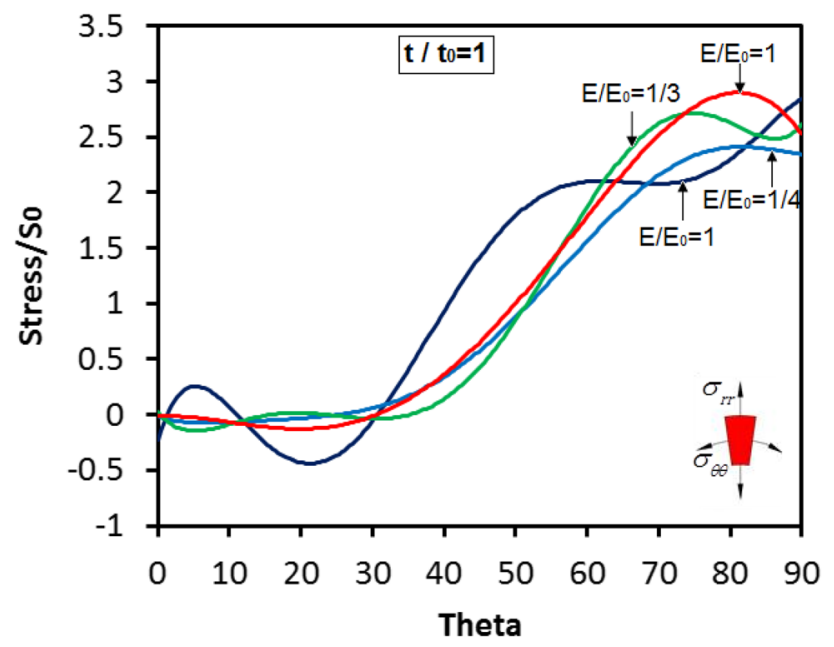

Fig. 15 Longitudinal stress around holes in the host plate for for Rs $\leq 1$

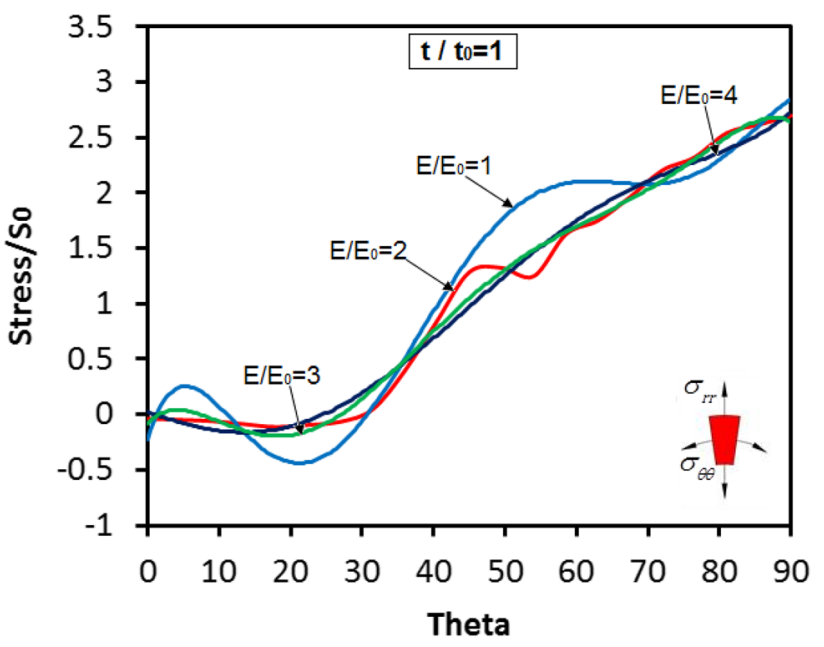

Fig. 16 Longitudinal stress around holes in the host plate for Rs $\geq 1$ 


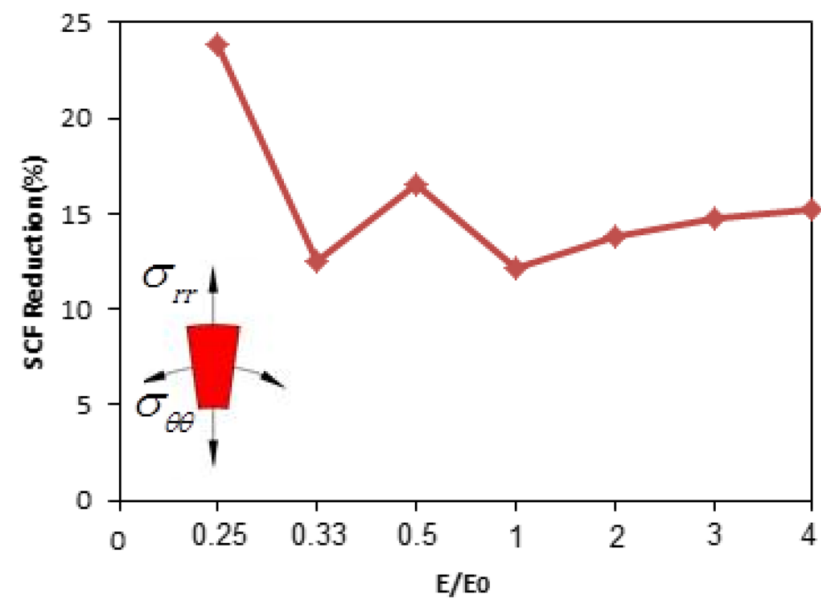

Fig. 17 Maximum stress concentration reduction in the host plate for different Rs

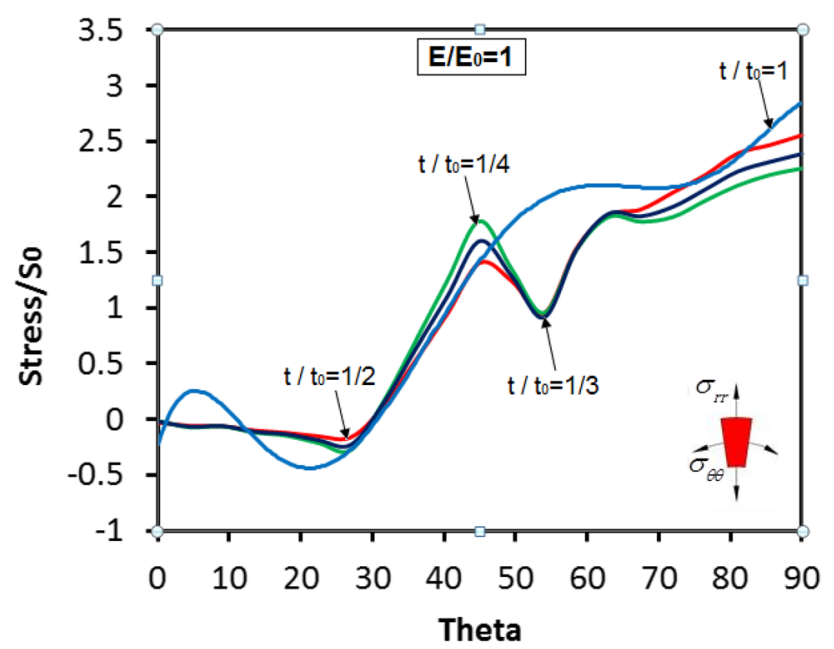

Fig. 18 Longitudinal stress around holes in the host plate for Rt $\leq 1$

ratio, two strain gauges are bounded at the top/bottom of the hole in a high stress concentration area. Figure 21 shows the schematic of the experimental tests.

The strain obtained from the data logger in the experimental test and FE analysis is presented in the Table 2. As this table shows, the difference between results from tests and $\mathrm{FE}$ analysis is in an acceptable range. The results show that in all stiffness ratios and thickness ratios, strains from experimental tests have less value than strains from $\mathrm{FE}$ results. One reason may be the effect of the adhesion in gluing piezoelectric patches to the host plate. Also, it is possible that the strain gauges were attached inaccurately on the top and bottom of the hole.

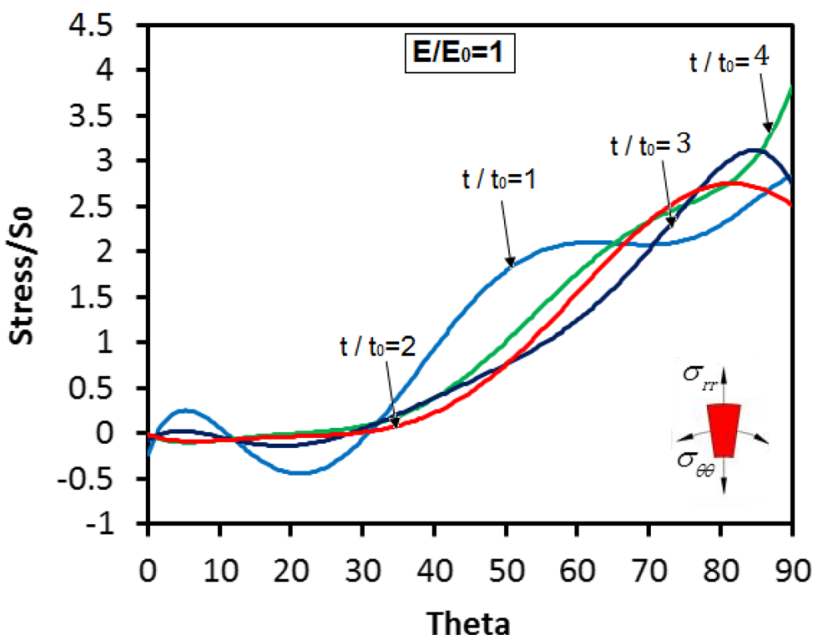

Fig. 19 Longitudinal stress around holes in the host plate for Rt $\geq 1$

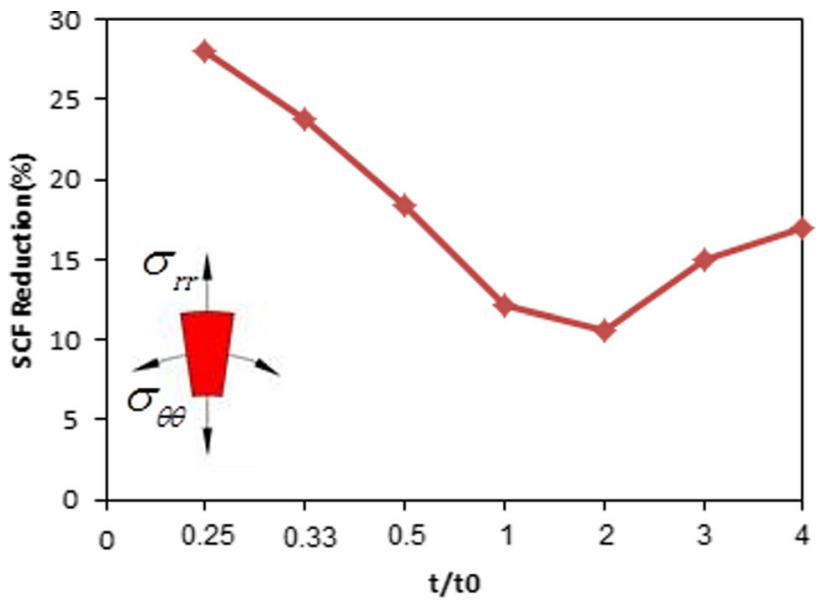

Fig. 20 Maximum stress concentration reduction in the host plate for different Rt

\section{Conclusion}

In this paper, two methods for reducing the stress concentration factor are considered. In the first method, the reduction in the stress concentration factor is affected directly by inducing positive strain to the host plate using piezoelectric patches. In the second method, by inducing negative strain, the stress line in the host plate is redistributed and the stress concentration factor is reduced. The results show that the stiffness ratio and thickness ratio of the host plate and piezoelectric patches affect the best location of actuators to reach the maximum reduction in stress concentration factor. Some experimental tests are 
(a)

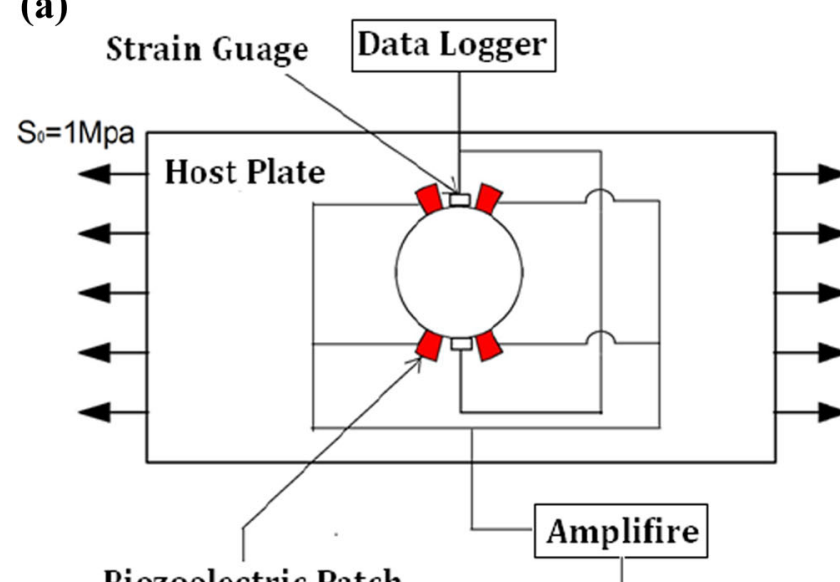

Piezoelectric Patch (b)

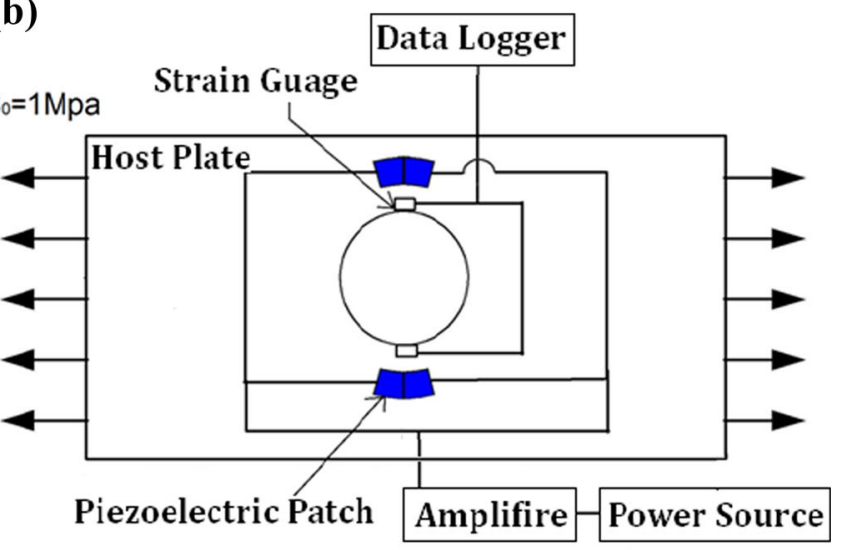

Power Source

Fig. 21 Schematic of experimental tests, a positive strain, b negative strain

Table 2 Comparison of the results of the test and analysis

\begin{tabular}{lllll}
\hline Condition & Volt & Strain from analysis $\times 10^{-6}$ & Strain from test $\times 10^{-6}$ & Error $(\%)$ \\
\hline Rs $=1 / 2$ & 0 & 62.2 & 54.7 & 12.1 \\
& 10 & 57.8 & 51.7 & 10.5 \\
& 20 & 47.4 & 42.7 & 9.9 \\
& 30 & 40.1 & 36.9 & 8 \\
& 40 & 32.8 & 30.5 & 7 \\
$\mathrm{Rt}=2$ & 0 & 60.9 & 54.8 & 10 \\
& 10 & 54.7 & 49.5 & 9.5 \\
& 20 & 48.3 & 44.4 & 8.1 \\
& 30 & 42 & 39.3 & 6.4 \\
& 40 & 35.7 & 33.6 & 5.8 \\
\hline
\end{tabular}

applied to validate the results. The results show good agreement between the FE analysis and experimental tests.

Open Access This article is distributed under the terms of the Creative Commons Attribution 4.0 International License (http://creative commons.org/licenses/by/4.0/), which permits unrestricted use, distribution, and reproduction in any medium, provided you give appropriate credit to the original author(s) and the source, provide a link to the Creative Commons license, and indicate if changes were made.

\section{References}

Chee KCY, Tong L, Steven GP (2002) Static shape control of composite plates using a slope-displacement-based algorithm. AIAA J 40:1611-1618

Da Mota Silva S, Ribeiro R, Rodrigues JD, Vaz M, Monteiro J (2004) The application of genetic algorithms for shape control with piezoelectric patches-an experimental comparison. Smart Mat Struct 13:220

Franco Correia VM, Mota Soares CM, Mota Soares CA (2003) Buckling optimization of composite laminated adaptive structures. Comp Struct 62:315-321
Hsu JC, Tseng CT, Chen YS (2014) Analysis and experiment of selffrequency-tuning piezoelectric energy harvesters for rotational motion. Smart Mat Struct 23:075013

Huang B, Kim HS (2014) Control of free-edge interlaminar stresses in composite laminates using piezoelectric actuators. Smart Mat Struct 23:074002

Jadhav PA, Bajoria AKM (2013) Free and forced vibration control of piezoelectric FGM plate subjected to electro-mechanical loading. Smart Mat Struct 22:065021

Kang Z, Tong L (2008) Topology optimization-based distribution design of actuation voltage in static shape control of plates. Comput Struct 86:1885-1893

Kang Z, Wang X, Luo Z (2012) Topology optimization for static shape control of piezoelectric plates with penalization on intermediate actuation voltage. J Mech Des 134:051006

Kumar R, Mishra B, Jain S (2008) Static and dynamic analysis of smart cylindrical shell. Finite Elem Anal Des 45:13-24

Kurata M, Li X, Fujita K, Yamaguchi M (2013) Piezoelectric dynamic strain monitoring for detecting local seismic damage in steel buildings. Smart Mat Struct 22:115002

Lin JC, Nien M (2007) Adaptive modeling and shape control of laminated plates using piezoelectric actuators. J Mat Proc Tech 189:231-236

Mehrabian AR, Yousefi-koma A (2011) A novel technique for optimal placement of piezoelectric actuators on smart structures. J Franklin Inst 348:12-23 
Nakasone P, Silva E (2010) Dynamic design of piezoelectric laminated sensors and actuators using topology optimization. J Intel Mat Sys Struct 21:1627-1652

Nguyen Q, Tong L (2004) Shape control of smart composite plate with non-rectangular piezoelectric actuators. Compos Struct 66:207-214

Nguyen Q, Tong L (2007) Voltage and evolutionary piezoelectric actuator design optimisation for static shape control of smart plate structures. Mat Des 28:387-399

Nguyen Q, Tong L, Gu Y (2007) Evolutionary piezoelectric actuators design optimisation for static shape control of smart plates. Comp Meth Appl Mech Eng 197:47-60

Platz R, Stapp C, Hanselka H (2011) Statistical approach to evaluating active reduction of crack propagation in aluminum panels with piezoelectric actuator patches. Smart Mat Struct 20:085009

Qing G, Qiu J, Liu Y (2006) A semi-analytical solution for static and dynamic analysis of plates with piezoelectric patches. Int J Solid Struct 43:1388-1403

Quintero AV, Besse N, Janphuang P, Lockhart R, Briand D, De Rooij NF (2014) Design optimization of vibration energy harvesters fabricated by lamination of thinned bulk-PZT on polymeric substrates. Smart Mat Struct 23:045041

Rafiee M, He X, Liew K (2014) Nonlinear analysis of piezoelectric nanocomposite energy harvesting plates. Smart Mat Struct 23:065001
Roy T, Chakraborty D (2009) Optimal vibration control of smart fiber reinforced composite shell structures using improved genetic algorithm. J Sound Vib 319:15-40

Sensharma PK, Haftka RT (1996) Limits of stress reduction in a plate with a hole using piezoelectric actuators. J Intel Mat Syst Struct 7:363-371

Sensharma PK, Palantera MJ, Haftka RT (1993) Stress reduction in an isotropic plate with a hole by applied induced strains. J Intell Mat Syst Struct 4:509-518

Shah D, Joshi S, Chan W (1994) Stress concentration reduction in a plate with a hole using piezoceramic layers. Smart Mat Struct $3: 302$

Sridharan S, Kim S (2009) Piezo-electric control of stiffened panels subject to interactive buckling. Int J Solid Struct 46:1527-1538

Wang Q, Wu N (2012) A review on structural enhancement and repair using piezoelectric materials and shape memory alloys. Smart Mat Struct 21:013001

Wu N, Wang Q (2010) Repair of vibrating delaminated beam structures using piezoelectric patches. Smart Mat Struct 19:035027

Wu N, Wang Q (2011) An experimental study on the repair of a notched beam subjected to dynamic loading with piezoelectric patches. Smart Mat Struct 20:115023

Zhang H, Lennox B, Goulding PR, Leumg AY (2000) A floatencoded genetic algorithm technique for integrated optimization of piezoelectric actuator and sensor placement and feedback gains. Smart Mat Struct 9:552 